\title{
Numerical Computation Based on the Method of Fundamental Solutions for a Cauchy Problem of Heat Equation
}

\author{
Ruihua Cao*

\begin{abstract}
School of Mathematics and Computer science, Shanxi Normal University, Linfen, China
\end{abstract} \\ *Corresponding author: caoruihua0056@126.com
}

Received May 03, 2014; Revised June 10, 2014; Accepted June 19, 2014

\begin{abstract}
In this note, a boundary integral equation method coupled with the method of fundamental solutions for solving an inverse heat conduction problem is considered. The Tikhonov regularization method is employed for solving this system of equations. Determination of regularization parameter is based on GCV criterion. To illustrate our main results, some numerical examples are given.
\end{abstract}

Keywords: inverse problem of the heat equation, method of fundamental solutions, integral equation method

Cite This Article: Ruihua Cao, "Numerical Computation Based on the Method of Fundamental Solutions for a Cauchy Problem of Heat Equation." Turkish Journal of Analysis and Number Theory, vol. 2, no. 3 (2014): 70-74. doi: 10.12691/tjant-2-3-3.

\section{Introduction}

In this article, we consider a Cauchy problem of heat equation, that is, determining the unknown temperature and heat flux at an inaccessible boundary from scattered temperature measurements on an accessible boundary or in some interior locations. This method is similar to the boundary control approach proposed by Leevan Ling and Tomoya Takeuchi in [1] where the authors considered a Cauchy problem for the Laplace equation. We use the standard integral equation method coupled with the method of fundamental solutions to solve the Cauchy problem for heat equation.

This kind of inverse heat conduction problem arises in some industrial and engineering applications, such as crystal growing [2] and material structure [3]. The Cauchy problem of heat equation is a highly ill-posed problem, because the solution does not depend continuously on the boundary date, ie, any small change on the input data can result in a dramatic change to the solution. So it is difficult to obtain an accurate and stable approximate solution. Usually one regularization strategy is necessary. In order to solve such problem, one can employ the boundary element method (BEM) [4], finite difference method(FDM) [5], finite element method(FEM) [6], and so on. Among these methods, the FDM and the FEM depend critically on the quality of mesh. However, generating a good quality mesh for complicated geometries could be time-consuming. Using the BEM can reduce the computational time and storage requirement but the problem of numerical in stability still persists.

Recently, several meshless and integration-free methods have been proposed. One of the most commonly used technique is the method of fundamental solutions. Hon and Wei have already successfully applied this method to solve One-dimensional and multidimensional inverse heat conduction problems in $[7,8]$. In this paper, the difference from one method in $[7,8]$ is that we use the method of fundamental solutions to solve a sequence of direct problems instead of solving the inverse problem directly.

\section{The Formulation of Problem and a Numerical Method}

The formulation of considered problem is

$$
\left\{\begin{array}{cl}
u_{t}=u_{x x}, & 0<x<1,0<t<t_{\max }, \\
u(x, 0)=u_{0}(x) & 0 \leq x \leq 1, \\
u(1, t)=f(t), & 0 \leq t \leq t_{\max }, \\
\frac{\partial u}{\partial x}(1, t)=g(t) & 0 \leq t \leq t_{\max } .
\end{array}\right.
$$

Where $t_{\max }$ is a given positive constant, $u_{0}(x), f(t)$ and $g(t)$ are given functions. Our aim is to compute the temperature and heat flux on the end $x=0$.

Let $w(x, t)$ be the solution of following forward problem

$$
\left\{\begin{array}{cl}
w_{t}=w_{x x}, & 0<x<1,0<t<t_{\max }, \\
w(x, 0)=u_{0}(x) & 0 \leq x \leq 1, \\
w(0, t)=0, & 0 \leq t \leq t_{\max }, \\
\frac{\partial w}{\partial x}(1, t)=g(t) & 0 \leq t \leq t_{\max } .
\end{array}\right.
$$


and define an operator $A: \phi(t) \rightarrow v(1, t)$ where $v(x, t)$ is the solution of the following forward problem

$$
\left\{\begin{array}{cl}
v_{t}=v_{x x}, & 0<x<1,0<t<t_{\max }, \\
v(x, 0)=0 & 0 \leq x \leq 1, \\
v(0, t)=\phi(t), & 0 \leq t \leq t_{\max }, \\
\frac{\partial v}{\partial x}(1, t)=0 & 0 \leq t \leq t_{\max } .
\end{array}\right.
$$

If we take $\phi:=u(0, t)$, then we know it satisfies the following operator equation

$$
A \phi=f(t)-w(1, t)
$$

In the following, we propose a numerical method based on the method of fundamental solutions to solve (4).

Find an approximate solution $\phi_{r}(t) \in V_{r}=\operatorname{span}\left\{1, t, t^{2}, \cdots t^{r-1}\right\}$ by a collocation method such that

$$
A \phi_{r}\left(t_{i}^{*}\right)=f\left(t_{i}^{*}\right)-w\left(1, t_{i}^{*}\right), i=1,2, \cdots n
$$

Where $\phi_{r}=\sum_{j=1}^{r} \beta_{j} t^{j-1}$, and $\left\{t_{i}^{*}\right\}$ is a set of collocation points on $\left[0, t_{\max }\right]$ and will be given in the following.

Note that the operate $A$ is linear, therefore we have $A \phi_{r}=\sum_{j=1}^{r} \beta_{j} A t^{j-1}$, Where $A t^{j}$ is determined by solving a direct problem (3) with $\phi=t^{j}$.

In the following we use the method of fundamental solutions to solve the forward problems (2) and (3). The fundamental solution of heat equation is

$$
F(x, t)=-\frac{1}{2 \sqrt{\pi t}} e^{\frac{-x^{2}}{4 t}} H(t)
$$

Where $H(t)$ is the Heaviside function. Assume that $T>t_{\max }$ is a constant, then the following function $G(x, t)=F(x, t+T)$ is a general solution of heat equation in the domain $[0,1] \times\left[0, t_{\max }\right]$.

Choose the collocation points

$$
\begin{aligned}
& \left\{\left(x_{j}, t_{j}\right) \mid j=1,2, \cdots l\right\} \subset[0,1] \times[0] \\
& \left\{\left(x_{l+j}, t_{l+j}\right) \mid j=1,2, \cdots m\right\} \subset[0] \times\left[0, t_{\max }\right] \\
& \left\{\left(x_{l+m+j}, t_{l+m+j}\right) \mid j=1,2, \cdots n\right\} \subset[1] \times\left[0, t_{\max }\right] .
\end{aligned}
$$

approximate solution of (3) with $\phi=t^{i-1}$, then $A t^{i-1}=v_{i}(1, t), i=1,2, \cdots n$. By using the initial and boundary conditions of (3), we know the unknown coefficients satisfy $A \lambda^{i}=b^{i}, i=1,2, \cdots n$, here

$$
A=\left[\begin{array}{c}
G\left(x_{k}-x_{j}, t_{k}-t_{j}\right) \\
G\left(x_{l+p}-x_{j}, t_{l+p}-t_{j}\right) \\
\frac{\partial G}{\partial x}\left(x_{l+m+s}-x_{j}, t_{l+m+s}-t_{j}\right)
\end{array}\right],
$$

$$
\begin{aligned}
& b^{i}=\left[\begin{array}{c}
0 \\
t_{l+p}^{i-1} \\
0
\end{array}\right], \lambda^{i}=\left[\begin{array}{c}
\lambda_{k}^{i} \\
\lambda_{l+p}^{i} \\
\lambda_{l+m+s}^{i}
\end{array}\right], \\
& k=1,2, \cdots l, p=1,2, \cdots m, \\
& s=1,2, \cdots n, j=1,2, \cdots l+m+n .
\end{aligned}
$$

Therefore $\lambda=\left[\lambda^{1}, \lambda^{2}, \cdots \lambda^{n}\right]=A^{+} B$ Where + denotes the pseudo inverse and $B=\left[b^{1}, b^{2}, \cdots b^{n}\right]$.

By similar procedure, we can obtain an MFS solution for (2) as $w(x, t)=\sum_{j=1}^{l+m+n} \alpha_{j} G\left(x-x_{j}, t-t_{j}\right)$ Where the coefficients $\alpha=\left[\alpha_{1}, \alpha_{2}, \cdots \alpha_{l+m+n}\right]^{T}$ satisfy $A \alpha=b$ and $b=\left[\begin{array}{c}u_{0}\left(x_{k}\right) \\ 0 \\ g\left(t_{l+m+s}\right)\end{array}\right]$ for $k=1,2, \cdots l, \quad s=1,2, \cdots n$.

By (5) with $t_{i}^{*}=t_{l+m+i}$, we know

$\sum_{i=1}^{r} \beta_{i} v_{i}\left(1, t_{l+m+s}\right)=f\left(t_{l+m+s}\right)-w\left(1, t_{l+m+s}\right), s=1,2, \cdots n(6)$

These leads to $M \lambda \beta=f-M \alpha, \quad$ in which $\beta=\left[\begin{array}{c}\beta_{1} \\ \cdot \\ \cdot \\ \cdot \\ \beta_{n}\end{array}\right], f=\left[\begin{array}{c}f\left(t_{l+m+1}\right) \\ \cdot \\ \cdot \\ \cdot \\ f\left(t_{l+m+n}\right)\end{array}\right]$ and

$M=\left[\begin{array}{c}G\left(x_{l+m+1}-x_{j}, t_{l+m+1}-t_{j}\right) \\ \cdot \\ \cdot \\ \cdot \\ G\left(x_{l+m+n}-x_{j}, t_{l+m+n}-t_{j}\right)\end{array}\right]$

$j=1,2, \cdots l+m+n$.

Denote $D=M \lambda$ and $\gamma=f-M \alpha$, then we need to solve the following linear system of equations

$$
D \beta=\gamma \text {. }
$$

In practical application, we can only get the measurement data $f^{\delta}, g^{\delta}$ of $f$ and $g$ which are usually contaminated by inherent measurement errors. Suppose that $\left|f^{\delta}(t)-f(t)\right| \leq \delta \forall t \in\left[0, t_{\max }\right]$,

$$
\left|g^{\delta}(t)-g(t)\right| \leq \delta \forall t \in\left[0, t_{\max }\right] .
$$

For the noisy data $f^{\delta}$ and $g^{\delta}$, the vector $\gamma$ becomes a noisy vector $\gamma^{\delta}$, we have to solve a ill-conditioned linear system

$$
D \beta=\gamma^{\delta} .
$$

Here, we adapt the Tikhonov regularized technique [9] to solve equations (8). The Tikhonov regularized solution 
to (8) is defined as the minimize $\beta_{\alpha}^{\delta}$ of the following Tikhonov functional $J(\beta)=\left\|D \beta-\gamma^{\delta}\right\|^{2}+\alpha^{2}\|\beta\|^{2}$, Where $\|\cdot\|$ denote the usual Euclidean norm and $\alpha>0$ is a regularized parameter.

The determination of a suitable regularization parameter is crucial to the accuracy of the regularized solution. In [10], the authors conclude that the GCV and L-curve choice rulers for Tikhonov regularization strategy are most effective. So in our computation we use the GCV method to determine a suitable value of $\alpha$. This method is to choose the regularization parameter $\alpha^{*}$ that minimizes the following GCV function $G(\alpha)=\frac{\left\|D \beta_{\alpha}^{\delta}-\gamma^{\delta}\right\|^{2}}{\left(\operatorname{trace}\left(I_{n}-D D^{I}\right)\right)^{2}}$, where $D^{I}$ is a matrix that produces the regularized solution when multiplied with $\gamma^{\delta}$, i.e. $\beta_{\alpha}^{\delta}=D^{I} \gamma^{\delta}$.

In our paper, we used the Matlab code developed by Hansen [11] based on SVD for solving the discrete illconditioned system (8). Denote the regularized solution to (8) by $\beta_{\delta}^{\alpha^{*}}$. The approximating solution to problem (4) is then given as

$$
\phi_{r}^{*}(t)=\sum_{i=1}^{r}\left(\beta_{\delta}^{\alpha^{*}}\right)_{i} t^{i-1}
$$

and the solution of (1) can be obtained by solving a direct problem using the MFS

$$
\left\{\begin{array}{cl}
u_{t}=u_{x x}, & 0<x<1,0<t<t_{\max }, \\
u(x, 0)=u_{0}(x) & 0 \leq x \leq 1 \\
u(0, t)=\phi_{r}^{*}, & 0 \leq t \leq t_{\max } \\
u(1, t)=f^{\delta}(t) & 0 \leq t \leq t_{\max }
\end{array}\right.
$$

\section{Numerical Examples}

In this section, we test several examples to show the effective of our proposed method. For simplicity, we assume that $t_{\max }=1$ and the noisy data $f_{i}^{\delta}=f_{i}+\delta f_{i} \operatorname{rand}(i)$ and $g_{i}^{\delta}=g_{i}+\delta g_{i} \operatorname{rand}(i)$, where $\delta$ indicates a relative noise level and $\operatorname{rand}(i)$ is a random number between [$1,1]$.

For evaluating numerical solutions, we compute a relative root mean squares error by the following formula $\operatorname{rel}(u)=\left(\sum_{i=1}^{N}\left(u\left(0, \overline{t_{i}}\right)-\phi_{r}^{*}\left(\overline{t_{i}}\right)\right)^{2} / \sum_{i=1}^{N} u\left(0, \overline{t_{i}}\right)^{2}\right)^{\frac{1}{2}}$, Where $\overline{t_{i}}$ is the test point and $N$ is the total number of test points on $\left[0, t_{\max }\right]$.

In the following numerical simulations, the number of collocation points is $l=m=n=11$ and the number of test points $N=55$ on $[0,1]$. The comparisons between the exact solutions and the approximation are given in figs $1-8$ and from these figures we can see that the numerical solution of the proposed method is effective for the Cauchy problem of heat equation. Our numerical solutions $\frac{\partial u}{\partial x}(0, t)$ are obtained by solving the direct problem of (11).

In our article, there have been many parameters, such as $T, r$ and source points $n_{s}=l+m+n$. In order to study the influence of these parameters on the numerical results. We are given the $\operatorname{rel}(u)$ and $\operatorname{rel}\left(u_{x}\right)$ with respect to various parameters in example 2 with $\delta=1 \%$.

Example 1. Let the exact solution for problem (1) be $u(x, t)=e^{-t}(\sin x+\cos x)$

All the given boundary data $f(t), g(t)$ and initial condition $u_{0}(x)$ can be obtained from the exact solution $u$.
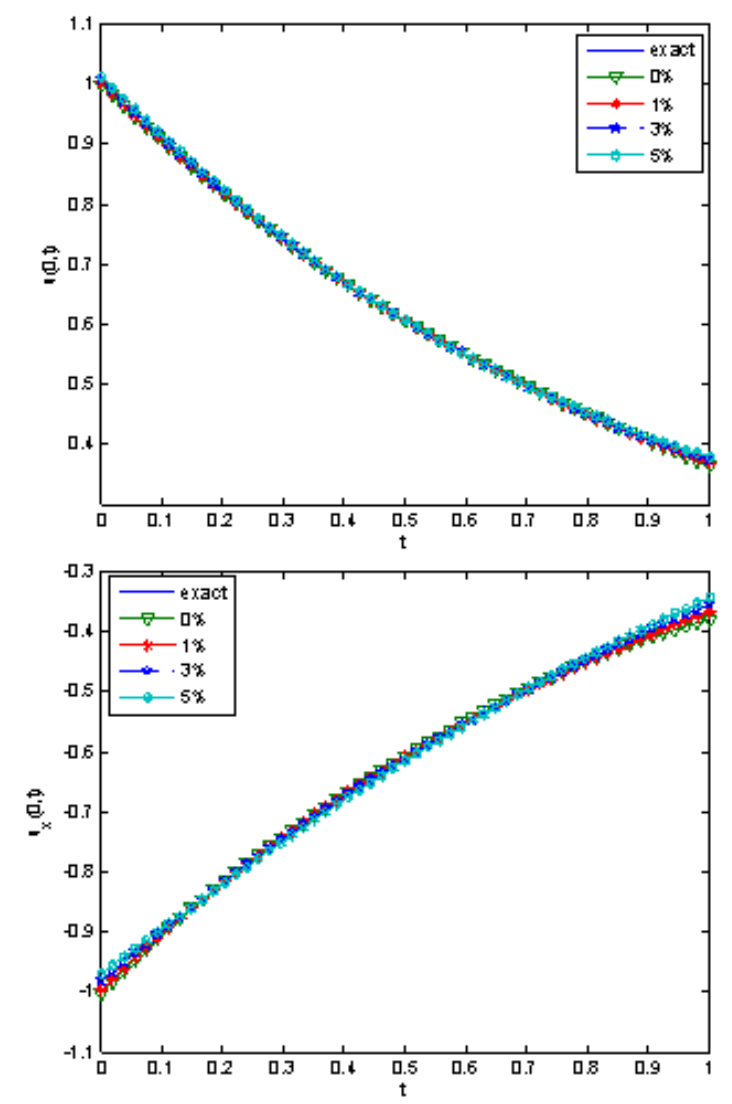

Figure 1. Example 1: The boundary temperature $u(0, t)$ and heat $u_{x}(0, t)$ with its numerical approximations and various noisy levels and $T=4, r=5$.

Table 1. Example 1: The relative root mean squares error $\operatorname{rel}(u(0, t))$ and $\operatorname{rel}\left(u_{x}(0, t)\right)$ for various values of $\delta$.

\begin{tabular}{ccc}
\hline$\delta$ & $\operatorname{rel}(u)$ & $\operatorname{rel}\left(u_{x}\right)$ \\
\hline 0 & 0.0012 & 0.0047 \\
\hline $1 \%$ & 0.0014 & 0.0025 \\
\hline $3 \%$ & 0.0051 & 0.0098 \\
\hline $5 \%$ & 0.0090 & 0.0176 \\
\hline
\end{tabular}

Example 2. Suppose that the exact solution $u$ is not available. The heat flux on the end $x=1$ can be obtained by solving the following forward problem 


$$
\left\{\begin{array}{l}
u_{t}=u_{x x}, \quad 0<x<1, \quad 0<t<1 \\
u(x, 0)=0 \quad 0 \leq x \leq 1 \\
-\frac{\partial u}{\partial x}(0, t)=\cos 5 t, \quad 0 \leq t \leq 1 \\
u(1, t)=t^{3} \quad 0 \leq t \leq 1
\end{array}\right.
$$

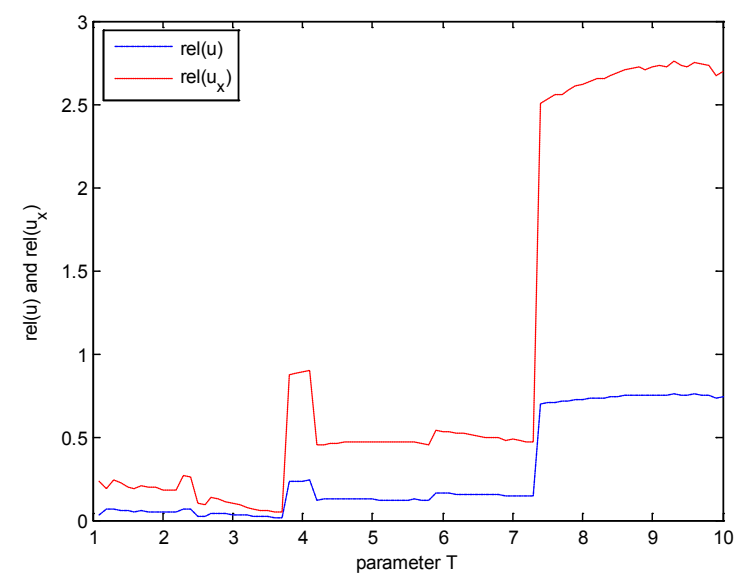

Figure 2. The relative root mean squares errors of solutions $u(0, t)$ and $u_{x}(0, t)$ with respect to $T$ for example 2 with noisy data $\delta=0.01$.

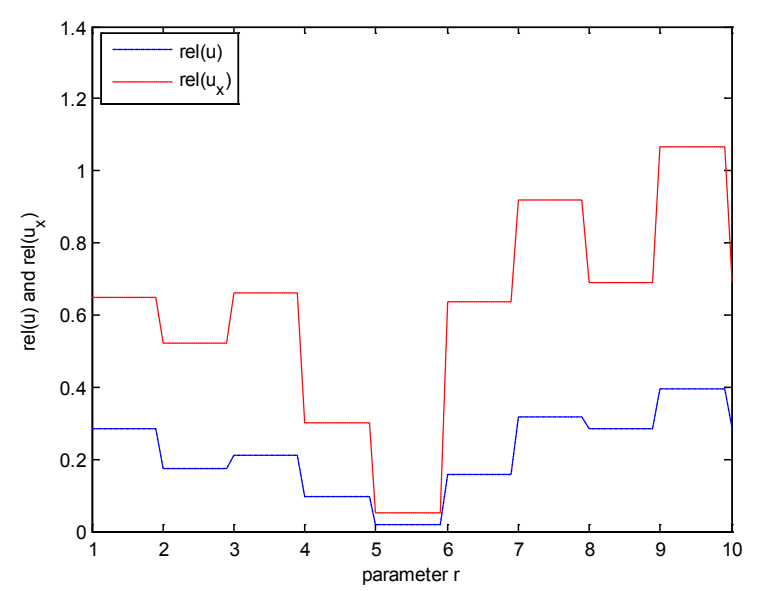

Figure 3. The relative root mean squares errors of solutions $u(0, t)$ and $u_{x}(0, t)$ with respect to $r$ for example 2 with noisy data $\delta=0.01$.

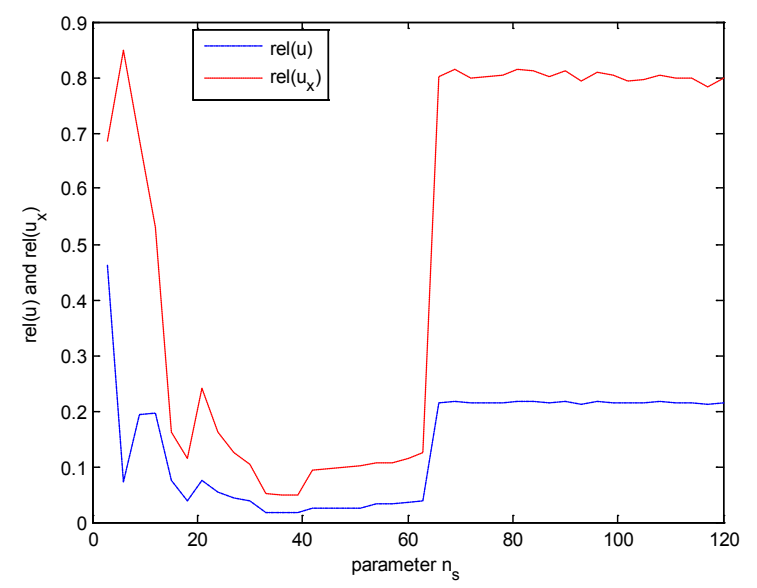

Figure 4. The relative root mean squares errors of solutions $u(0, t)$ and $u_{x}(0, t)$ with respect to $n_{s}$ for example 2 with noisy data $\delta=0.01$
Table 2. Example 2: The relative root mean squares error $\operatorname{rel}(u(0, t))$ and $\operatorname{rel}\left(u_{x}(0, t)\right)$ for various values of $\delta$.

\begin{tabular}{ccc}
\hline$\delta$ & $\operatorname{rel}(u)$ & $\operatorname{rel}\left(u_{x}\right)$ \\
\hline 0 & 0.0175 & 0.0505 \\
\hline $1 \%$ & 0.0179 & 0.0521 \\
\hline $3 \%$ & 0.0206 & 0.0592 \\
\hline $5 \%$ & 0.0237 & 0.0700 \\
\hline
\end{tabular}
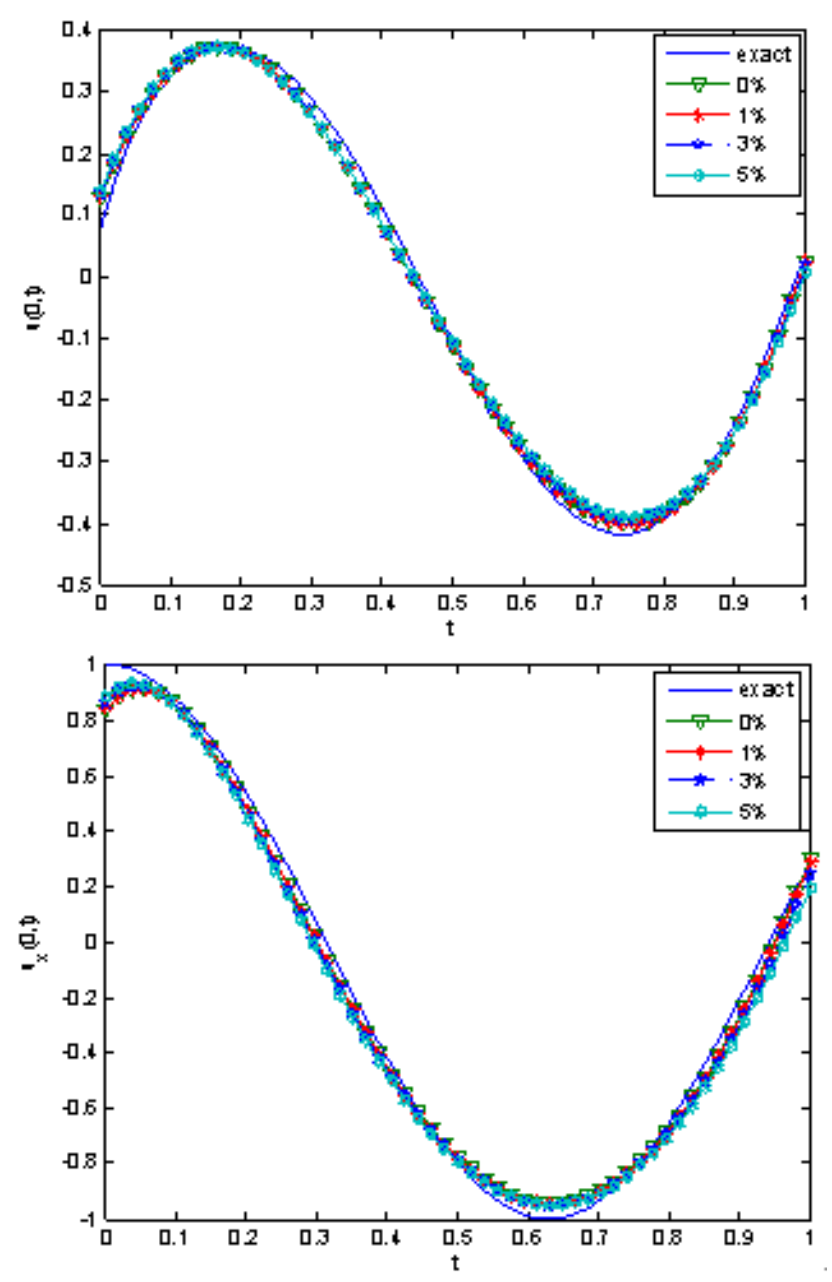

Figure 5. Example 2: The boundary temperature $u(0, t)$ and heat $u_{x}(0, t)$ with its numerical approximations and various noisy levels and $T=3.5, r=5$.

Example 3. Suppose that the exact solution $u$ is not available. The temperature on the surface $x=1$ can be calculated by solving the following forward problem

$$
\begin{cases}u_{t}=u_{x x} & 0<x<1,0<t<1, \\
u(x, 0)=x-1 & 0 \leq x \leq 1, \\
u(1, t)=0 & 0 \leq t \leq 1, \\
u(0, t)=\left\{\begin{array}{ccc}
-1 & t \in[0,0.5] & 0 \leq t \leq 1 \\
1 & t \in(0.5,1] & 0
\end{array}\right.\end{cases}
$$

Table 3. Example 3: The relative root mean squares error $\operatorname{rel}(u(0, t))$ and $\operatorname{rel}\left(u_{x}(0, t)\right)$ for various values of $\delta$.

\begin{tabular}{ccc}
\hline$\delta$ & $\operatorname{rel}(u)$ & $\operatorname{rel}\left(u_{x}\right)$ \\
\hline 0 & 0.3613 & 0.1556 \\
\hline $1 \%$ & 0.3609 & 0.1560 \\
\hline $3 \%$ & 0.3607 & 0.1572 \\
\hline $5 \%$ & 0.3613 & 0.1592 \\
\hline
\end{tabular}



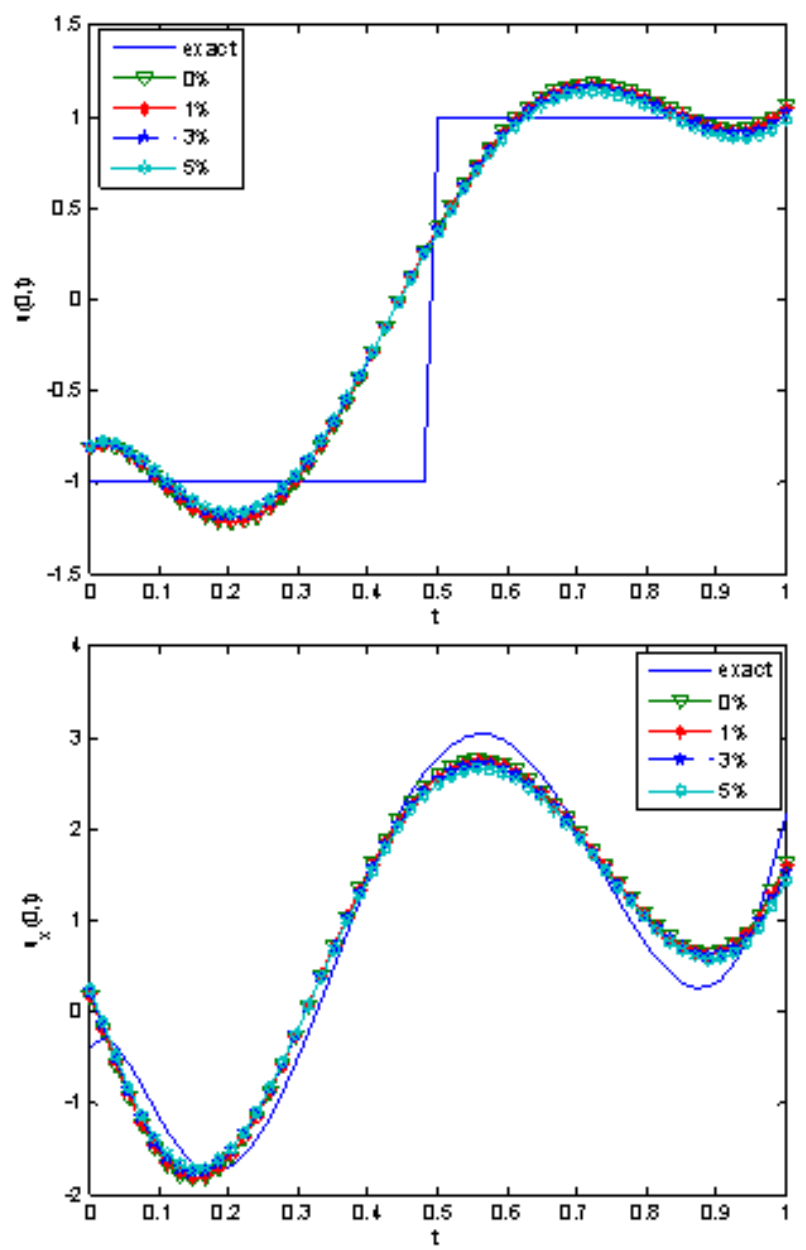

Figure 6. Example 3: The boundary temperature $u(0, t)$ and heat $u_{x}(0, t)$ with its numerical approximations and various noisy levels and $T=3.2 r=6$.

\section{Conclusion}

In this note, a Cauchy problem of heat equation is investigated by using a boundary integral equation method coupled with the method of fundamental solution, use of discrete Tikhonov regularization with generalized cross validation criterion for choosing a suitable regularization parameter stabilizes the resultant ill-conditioned system. Numerical examples with both known and unknown exact solutions are presented. The computed results show that our proposed method is reasonable, feasible and stable to this highly ill-posed inverse heat conduction problem.

\section{References}

[1] L. Ling, T. Takeuchi. Boundary control for inverse Cauchy problems of the Laplace equations. CMES Comput Model Eng Sci, 2008, Vol. 29, No. 1, pp. 45-54.

[2] M. C. Flemings. Solidification processing. McGraw-Hill, New York, 1974.

[3] D. A. Porter, K. E. Easteling. Phase Transformations in Metals and Alloys. Chapman Hall, London, 1981.

[4] D. Lesnic, L Elliott, D. B. Ingham. Application of the boundary element method to inverse heat conduction problems. International Communications in Heat and Mass Transfer, 1996 Vol. 39, No. 7, PP. 1503-1517.

[5] L Guo, D. Murio. A mollified space-marching finite-difference algorithm for the two-dimensional inverse heat conduction problem with slab symmetry. Inverse problem, 1991, Vol. 7, No. 2 PP. 247-259.

[6] T. R. Hsu, N. S. Sun, G.G. Chen, Z. L. Gong. Finite element formulation for two-dimensional inverse heat conduction analysis. Journal of Heat Transfer, 1992, Vol. 114. No. 3, PP. 553-557.

[7] T. Wei, Y. C. Hon. A fundamental solution method for inverse heat conduction problem. Engineering analysis with boundary elements, 2004, Vol. 28, No. 5, pp. 489-495.

[8] Y. C. Hon, T. Wei. The method of fundamental solution for solving multidimensional inverse heat conduction problems. CMES Compt. Model. Eng. Sci, 2005, Vol. 7, No. 2, pp. 119-132.

[9] A. N. Tikhonov, V. Y. Arsenin. On the solution of ill-posed problems. John Wiley and Sons, New York, 1977.

[10] T. Wei, Y. C. Hon, L. Ling. Method of fundamental solutions with regularization techniques for Cauchy problems of elliptic operators. Engineering Analysis with Boundary Elements, 2007, Vol. 31, No. 4, pp. 373-385.

[11] P. C. Hansen. Regularization Tools: a Matlab package for analysis and solution of discrete of ill-posed problems. Numerical Algorithms, 1994, Vol. 6, No. 1-2, PP. 1-35. 